\title{
Temporal Trends and Predictors of Pancreatitis Patients Who Leave Against Medical Advice: A Nationwide Analysis
}

\author{
Fahad Chaudhary ${ }^{\mathrm{a}, \mathrm{d}, \mathrm{e}}$, Ridwaan Albeiruti ${ }^{\mathrm{a}, \mathrm{d}}$, Fahad Alqahtani ${ }^{\mathrm{b}}$, Mohamed Alhajji ${ }^{\mathrm{b}}$, \\ Nathan Lerfald ${ }^{a}$, William Hutson ${ }^{\mathrm{c}}$
}

\begin{abstract}
Background: Acute pancreatitis is the leading gastrointestinal cause of hospital admissions. Our study aims to determine the trends and predictors of discharge against medical advice (AMA).

Methods: We utilized the Nationwide Inpatient Sample (2003 - 2016) to identify patients admitted with pancreatitis. We compared in-hospital complications and determined predictors of discharge AMA using a multivariate logistic regression.
\end{abstract}

Results: A total of 7,158,894 patients were admitted with pancreatitis. Of those, 199,351 left AMA. Discharge AMA increased over time from $2.3 \%$ to $3.2 \%$. Patients who left AMA were more likely to be younger, male, black, and a lower socioeconomic status (SES). They had a greater prevalence of depression, cirrhosis, smoking, drug abuse, and human immunodeficiency virus (HIV) infection. Alcohol use was the most likely etiology of pancreatitis among those leaving AMA. In a multivariate regression, patients more likely to leave AMA included: age 18 - 44, male, and black. Patients with a history of depression, drug abuse, and HIV infection were also more likely to be discharged AMA.

Conclusions: Discharges AMA increased over time. Predictors of AMA include patients who are younger, male, black, lower socioeconomic status, and have a history of depression, HIV infection, alcohol and drug use. Future studies are necessary to examine the reasons for discharge AMA among this population.

Keywords: Pancreatitis; Discharge; Against medical advice; Predictors

Manuscript submitted February 21, 2020, accepted March 4, 2020

aDepartment of Medicine, West Virginia University, Morgantown, WV, USA ${ }^{b}$ Division of Cardiology, West Virginia University, Morgantown WV, USA ${ }^{\mathrm{c} S e c t i o n}$ of Digestive Diseases, West Virginia University, Morgantown, WV, USA

dThey contributed equally to the manuscript.

${ }^{e}$ Corresponding Author: Fahad Chaudhary, Department of Medicine, West Virginia University School of Medicine, 1 Medical Center Drive, Morgantown, WV 26505-8059, USA. Email: chaudharyff@gmail.com

doi: https://doi.org/10.14740/gr1272

\section{Introduction}

Acute pancreatitis (AP) is the leading gastrointestinal cause of hospital admissions, accounting for more than 275,000 admissions per year in the USA $[1,2]$. Up to $75 \%$ of patients that present with AP to the emergency department require admission, which leads to an annual healthcare expenditure estimated at $\$ 2.5$ billion dollars [1-3]. Furthermore, nearly half of all readmissions are related to pancreatitis [4]. In the USA, alcohol and biliary disease are the most common causes of AP. With alcohol consumption in the USA on the decline in recent years, gallstone-related causes of pancreatitis are increasing, presumably due to a rise in obesity rates and an aging population $[5,6]$.

Nearly $0.6 \%$ of all hospitalizations result in discharge against medical advice (AMA) [7-10]. Historically, patients with low socioeconomic status (SES), a history of substance abuse, and mental illness have been found to have the highest rates of AMA discharges [7, 10-15]. The incidence and predictors of AMA discharge have been previously studied in patients with human immunodeficiency virus (HIV) infection, asthma, acute myocardial infarction, and inflammatory bowel disease (IBD), however this topic is yet to be studied in patients with pancreatitis. Patients with pancreatitis often have a wide variety of symptoms that are difficult to manage, which may lead to patient dissatisfaction. Leaving AMA results in incomplete treatment, increased rate of readmission, and increased health care utilization $[7,14]$. Our study aims to determine the prevalence and trends of discharge AMA in patients with pancreatitis. Additionally, we sought to assess the predictors associated with discharge AMA.

\section{Materials and Methods}

\section{Data source}

The Nationwide Inpatient Sample (NIS) of the Healthcare Cost and Utilization Project (HCUP) was used to derive patientrelevant information between January 1, 2003 and December 31,2016 . The NIS is the largest publicly available all-payer claims-based database that contains clinical and resource utilization information on patient discharges from approximately 


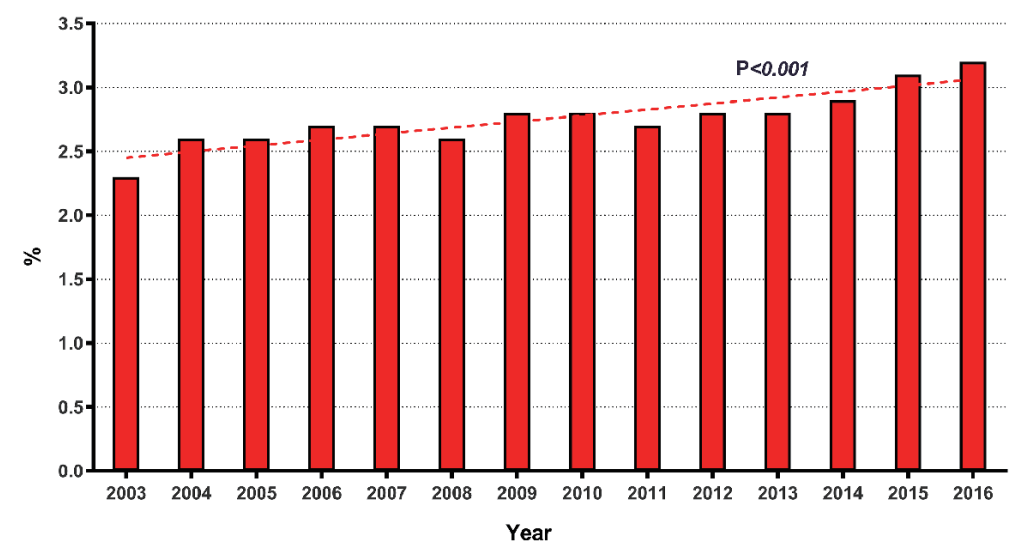

Figure 1. Trends of discharge AMA in patient admitted with acute pancreatitis (2003 - 2016). AMA: against medical advice.

1,000 non-federal hospitals in 46 states. These data are stratified to represent approximately $20 \%$ of US inpatient hospitalizations across different hospital and geographic regions (random sample). National estimates of the entire US hospitalized population were calculated using the Agency for Healthcare Research and Quality (AHRQ) and weighting methods. The study was exempt by the institutional review board because the NIS is a publicly available and de-identified database.

\section{Study sample}

Our study sample included patients aged 18 years or older who were hospitalized with a primary diagnosis of acute or chronic pancreatitis. We excluded patients with missing data on their discharge disposition, who were transferred out of the hospital, and those who died. Patients were identified using International Classification of Diseases-Ninth and Tenth Revision-Clinical Modification (ICD-9-CM and ICD-10-CM) codes (Supplementary Material 1, www.gastrores.org). The final sample included 7,158,894 pancreatitis hospitalizations.

\section{Study endpoints}

The primary outcome was disposition at discharge, specifically, whether a patient left the hospital AMA or was routinely discharged. Covariates included age, sex, race (white, black, Hispanic), insurance status (Medicare, Medicaid, private, or uninsured), income, bed size, weekend admission, and hospital location (urban, rural). In-hospital complications studied were urinary tract infection (UTI), acute kidney injury (AKI), acute dialysis requirement, septic shock, respiratory failure, length of stay (LOS), and cost.

\section{Statistical analysis}

Weighted national estimates were used in all statistical analyses. The Cochrane-Armitage test was used to assess the statistical significance of the temporal trend incidence of AMA discharge in patients admitted with pancreatitis. Patients were categorized into patients with pancreatitis who left AMA and those who were routinely discharged. Descriptive statistics were presented as frequencies with percentages for categorical variables. Mean, standard deviation, median, 25th and 75th percentiles were reported for continuous measures. Baseline characteristics were compared using Pearson Chi-squared test and Fisher's exact test for categorical variables and an independent-samples $t$-test for continuous variables. Univariate and multivariate logistic regression was performed to estimate odds ratios (ORs) with $95 \%$ confidence intervals (CIs) to determine significant predictors for discharge AMA. A type I error of $<0.05$ was considered statistically significant. To analyze monotonic trend data over time, the Mann-Kendall trend test was employed with significance level at $<5 \%$. All statistical analyses were performed with SPSS version 25 (IBM Corporation, Armonk, NY).

\section{Results}

Between 2003 and 2016, a total of 7,158,894 admissions for pancreatitis were identified in the NIS. Of those, 199,351 (2.8\%) left AMA. The incidence of discharge AMA has increased over time from 2.3\% in 2003 to $3.2 \%$ in $2016(\mathrm{P}<$ 0.001 ) (Fig. 1). Patients who left AMA were more likely to be younger $(49.4 \%$ vs. $5.8 \%, \mathrm{P}<0.001)$, male $(68.2 \%$ vs. $50.2 \%$, $\mathrm{P}<0.001)$, black $(24.4 \%$ vs. $17.5 \%, \mathrm{P}<0.001)$, and of a lower SES $(38.6 \%$ vs. $14.7 \%, \mathrm{P}<0.001)$ compared to those who were routinely discharged (Table 1). Patients who left AMA had a greater prevalence of depression $(10.6 \%$ vs. $7.1 \%, \mathrm{P}<$ $0.001)$, cirrhosis $(10.9 \%$ vs. $8.6 \%, \mathrm{P}<0.001)$, smoking $(44.4 \%$ vs. $22.6 \%, \mathrm{P}<0.001)$, drug abuse $(18.4 \%$ vs. $6.8 \%, \mathrm{P}<0.001)$, and HIV infection $(3.0 \%$ vs. $1.4 \%, \mathrm{P}<0.001)$. These patients' etiology of pancreatitis was more likely to be a result of alcohol use $(52.3 \%$ vs. $23.5 \%, \mathrm{P}<0.001)$ rather than biliary $(9.5 \%$ vs. $22.3 \%, \mathrm{P}<0.001)$ or other non-alcoholic causes $(16 \%$ vs. $26.6 \%, \mathrm{P}<0.001$ ) (Table 2). The trends in etiology of pancreatitis over the study time period is shown in Figure 2.

However, in-hospital complications including AKI, AKIrequiring hemodialysis, respiratory failure, and septic shock, 
Table 1. Baseline Characteristics of the Study Population

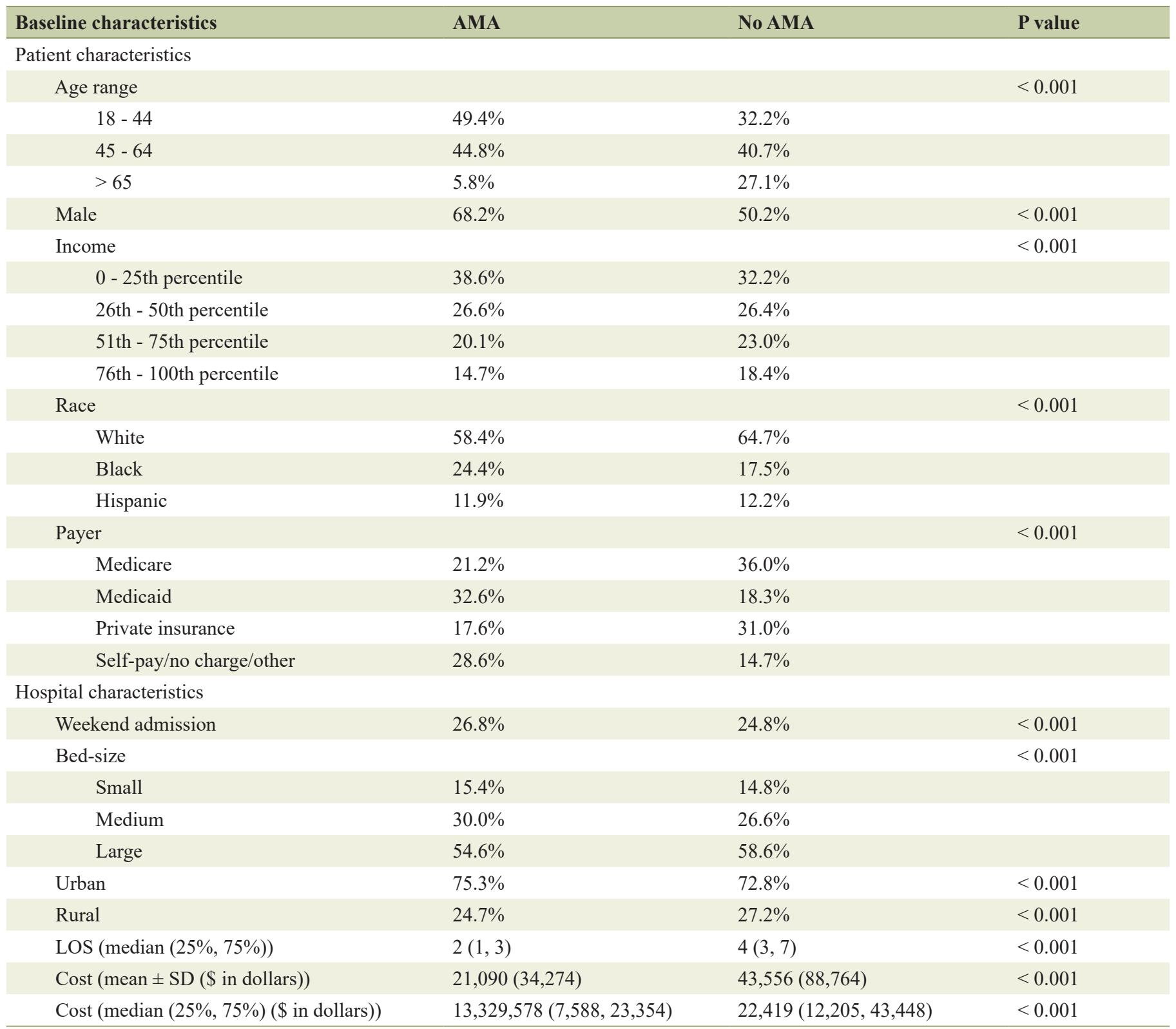

AMA: against medical advice; N: number; SD: standard deviation; LOS: length of stay.

and those receiving interventions such as endoscopic retrograde cholangiopancreatography (ERCP) were less frequently seen in patients who left AMA (Table 2).

In a multivariate logistic regression analysis, pancreatitis patients with the following characteristics were more likely to leave AMA: age 18 - 44 (OR: 4.14, 95\% CI: 4.04 - 4.24, P < 0.001 ), age 45 - 64 (OR: 3.21, 95\% CI: $3.14-3.29, \mathrm{P}<0.001$ ), male (OR: 1.58, 95\% CI: 1.56 - 1.60, P < 0.001), black (OR: 1.06, 95\% CI: $1.05-1.07, \mathrm{P}<0.05)$, and weekend admission (OR: 1.08, 95\% CI: $1.07-1.09, \mathrm{P}<0.001$ ). Patients with alcoholic pancreatitis were more likely to leave AMA (OR: 1.81, 95\% CI: $1.79-1.83, \mathrm{P}<0.001)$, than those with admissions secondary to biliary pancreatitis (OR: $0.85,95 \%$ CI: 0.84 -
$0.87, \mathrm{P}<0.001)$. Patients who had history of depression (OR: 1.05, 95\% CI: $1.03-1.07, \mathrm{P}<0.001$ ), drug abuse (OR: 1.44, 95\% CI: $1.42-1.46, \mathrm{P}<0.001$ ) and HIV infection (OR: 1.25, 95\% CI: $1.21-1.29, \mathrm{P}<0.001)$ were more likely to be discharged AMA (Table 3).

\section{Discussion}

This study describes the prevalence of discharge AMA among patients who were admitted with acute and chronic pancreatitis and evaluates associated predictors. Additionally, our study reports the trend of discharge AMA over time. To our knowl- 
Table 2. Patient Comorbidities, Complications and Interventions

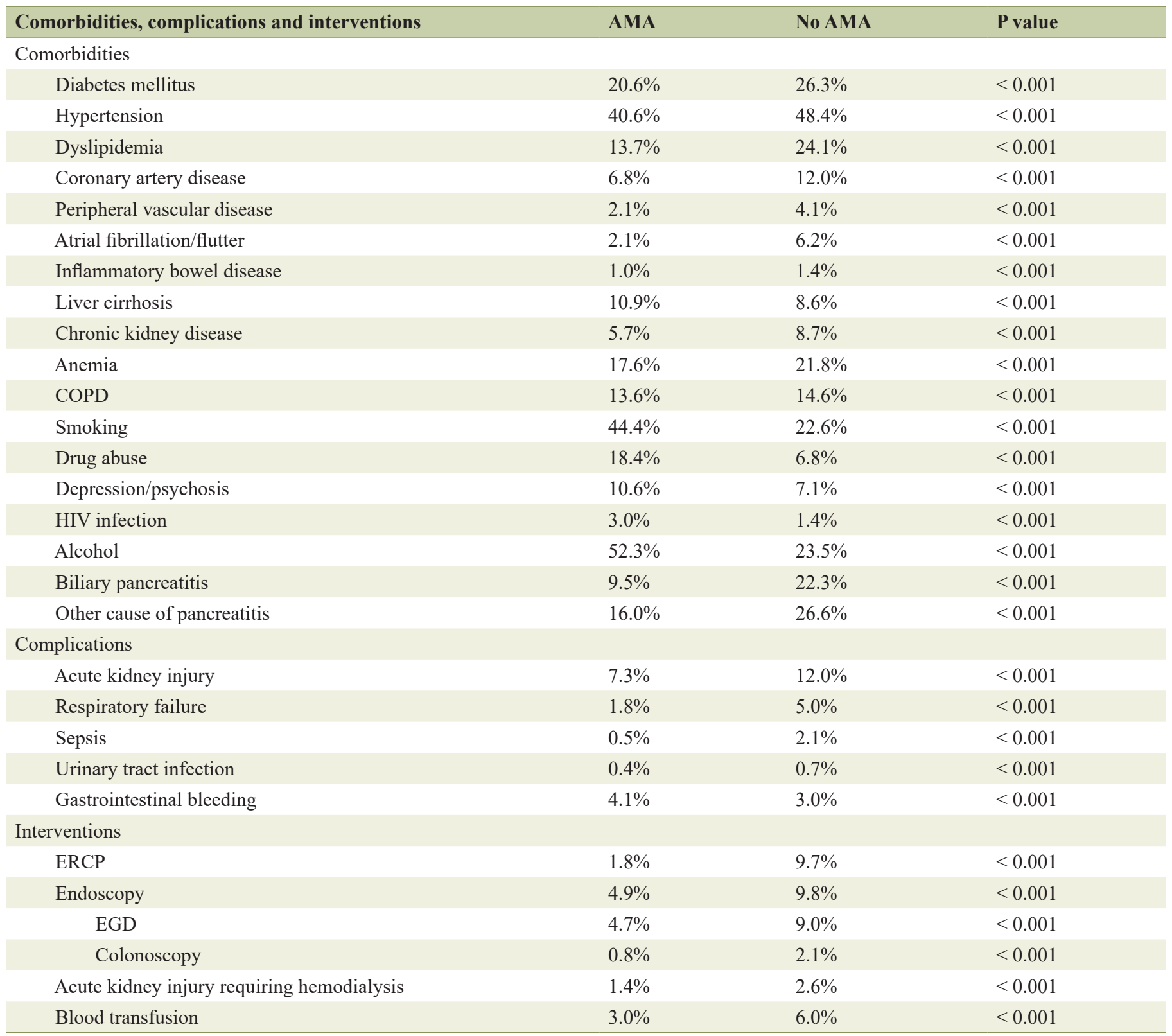

AMA: against medical advice; COPD: chronic obstructive pulmonary disease; HIV: human immunodeficiency virus; EGD: esophagogastroduodenoscopy; ERCP: endoscopic retrograde cholangiopancreatography.

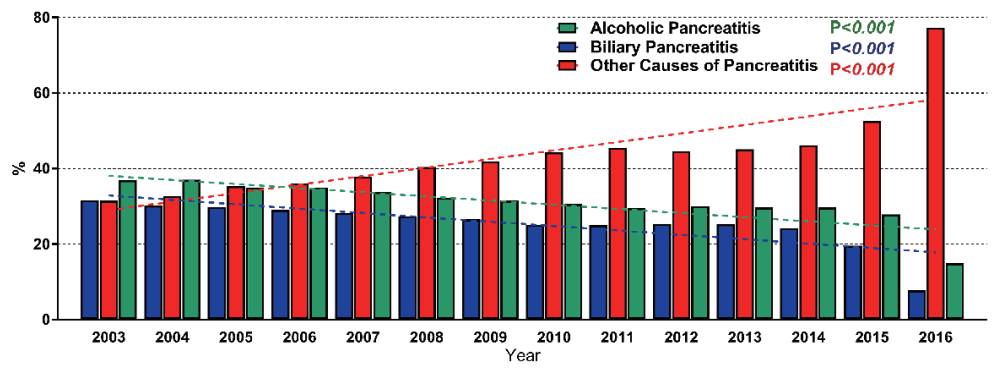

Figure 2. Trends in pancreatitis admissions overall and by etiology (alcohol, biliary, and other). 
Table 3. Multivariate Logistic Regression Analysis for Predictors of Discharge Against Medical Advice Among Patients Admitted With Pancreatitis

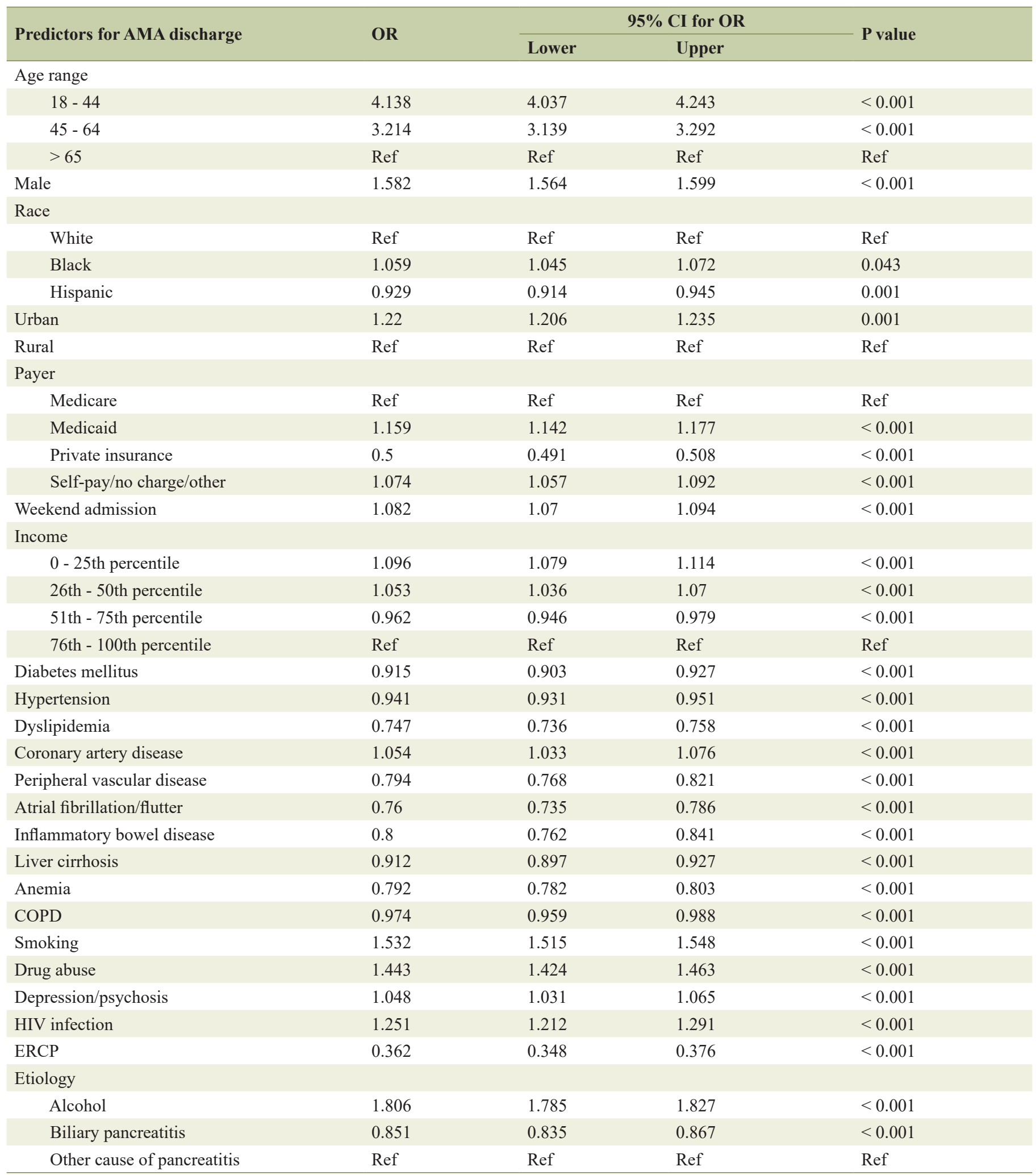

AMA: against medical advice; COPD: chronic obstructive pulmonary disease; HIV: human immunodeficiency virus; ERCP: endoscopic retrograde cholangiopancreatography; OR: odds ratio; Cl: confidence interval; Ref: reference. 
edge, this is the first and largest nationwide analysis to study AMA trends in pancreatitis patients. The main findings of our study were: 1) The number of discharges AMA increased over time; 2) Nearly 1 in 36 pancreatitis admissions leave AMA; 3) The predictors of AMA are patients that are younger, male, black, lower socioeconomic status, and have a history of depression, alcohol use, drug use, and HIV infection.

The trend of discharge AMA among patients admitted for pancreatitis has increased from 2003 - 2016 (Fig. 1). Despite improvements in the management of pancreatitis which has led to decreased mortality over time, our study shows that AMA rates have continued to increase [6]. This may be explained by patients' lack of insurance, higher financial burdens, poor education, and increased dependence on drugs and alcohol among this cohort.

We found a rate of AMA discharge to be $2.8 \%$, which is a notable increase compared to that of all-cause admissions $(1.2-1.4 \%)[10,16]$. Naturally, discharge AMA rates vary by admission diagnosis. AMA rates for general medicine services have been reported to be $0.6 \%$, while other conditions studied include: IBD $(1.3 \%)$, cirrhosis $(2.8 \%)$, pneumonia $(1.3 \%)$, and HIV infection (13\%) [16-19]. Comparatively, patients with a diagnosis of drug use carry a discharge AMA rate of $17 \%$, while those with alcohol use disorder were found to leave AMA at a rate of $14 \%[12,20]$. In patients with HIV infection, asthma, acute myocardial infarction, and IBD the predictors of AMA discharge include recent drug use, dissatisfaction with narcotic prescriptions, family obligations, male sex, and financial constraints in those with low socioeconomic status [7, 11, 21, 22].

In a multivariate analysis, our findings suggest that there are several patient and socioeconomic factors associated with discharge AMA in pancreatitis patients. These factors include: younger age, male sex, black, insured by Medicaid, and lower household income (Table 3). Pancreatitis patients between the ages of 18 - 44 were four times more likely to discharge AMA compared to elderly patients ( $>65$ years). Males also had a greater than 1.5-fold increased likelihood to discharge AMA. Those suffering from substance abuse with alcohol, drugs, and tobacco were nearly 1.5 times more likely to discharge AMA. Similarly, we also found socioeconomic status to have a bearing on the rate of discharge AMA [10, 11, 13, 14, 17]. These factors have been previously described as predictors for higher AMA rates in other populations [7, 10-15]. Based on these studies, patients of various diagnoses self-discharge for similar reasons, lending to the generalizability of our results. Interestingly, the etiology of pancreatitis was also predictive of whether a patient would leave AMA, such as those with alcohol as an etiology being approximately 1.8 times more likely to discharge AMA, while those with a biliary etiology were nearly 1.2 times less likely.

Understanding the reasons for patients discharging AMA is critical due to these patients having potentially worse outcomes and higher rates of readmission [13]. Patients are believed to discharge themselves AMA for a variety of reasons including personal or financial obligations, patient sense of improvement, lack of improvement, dissatisfaction with care, expectation of a shorter stay, underlying addiction with desire to use, and lack of a primary care physician [23-26]. Interestingly, studies have reported high-risk populations such as those with HIV infection and intravenous drug user (IVDU) to be less likely to leave AMA if they are receiving methadone or have social support from family and friends [27].

The LOS and cost of hospitalization in the AMA population was half that of patients who did not leave AMA (Table 1). Further studies are needed to determine the burden of AMA discharge in patients with pancreatitis who may present for a later readmission and with a more severe and complicated course.

Our study is not without limitations. First, the NIS is derived from hospital claims data and subject to the shortcomings of other administrative data sets. Inconsistencies related to over- or under-coding are possible, but AHRQ quality control measures should minimize those possibilities. Also, the ICD-9 codes used in our study have been used and/or validated in several prior studies [28-30]. Second, we used the principle diagnosis of pancreatitis to identify our study cohort. Hence, our data may not reflect the incidence or outcomes of pancreatitis among patients, who were admitted for another reason and developed pancreatitis during the hospitalization or were diagnosed with pancreatitis after admission. Moreover, due to the nature of the NIS database, each record accounts for a single hospitalization and not for an individual, which may lead to within-patient correlation. Third, NIS does not allow us to capture more granular information regarding the timing and severity of pancreatitis. Our data do not allow us to determine the reasons for discharge AMA, for example, differentiating between discharges due to inadequate symptom control and treatment versus dissatisfaction for other reasons (i.e. providers, hospital characteristics, etc.). Despite these limitations, we believe this study offers important insights into factors that may help prevent discharge AMA and therefore improve overall outcomes in patients with pancreatitis.

Future studies are necessary to examine the reasons for discharge AMA among this patient population. Improvements in treatment and symptoms control as well as identification of patients at high risk for discharge AMA may help to reduce self-discharge, readmissions, hospital costs, and subsequent morbidity.

\section{Supplementary Material}

Suppl 1. ICD9/10 Codes.

\section{Acknowledgments}

None to declare.

\section{Financial Disclosure}

None to declare.

\section{Conflict of Interest}

None of the authors has any conflict of interest to disclose. 


\section{Informed Consent}

Not applicable.

\section{Author Contributions}

FC, RA, and FA designed the study, acquired the database and performed the statistical analysis. FC, RA, FA, NL, and WH performed the background search and drafted the manuscript. WH supervised the study and made critical revisions of the manuscript. FC, RA, FA, and MA constructed all tables and figures.

\section{Data Availability}

The data supporting the findings of this study are available from the corresponding author upon reasonable request.

\section{References}

1. Forsmark CE, Vege SS, Wilcox CM. Acute pancreatitis. N Engl J Med. 2016;375(20):1972-1981.

2. Peery AF, Crockett SD, Barritt AS, Dellon ES, Eluri S, Gangarosa LM, Jensen ET, et al. Burden of Gastrointestinal, Liver, and Pancreatic Diseases in the United States. Gastroenterology. 2015;149(7):1731-1741 e1733.

3. McNabb-Baltar J, Ravi P, Isabwe GA, Suleiman SL, Yaghoobi M, Trinh QD, Banks PA. A population-based assessment of the burden of acute pancreatitis in the United States. Pancreas. 2014;43(5):687-691.

4. Shah R, Haydek C, Mulki R, Qayed E. Incidence and predictors of 30-day readmissions in patients hospitalized with chronic pancreatitis: A nationwide analysis. Pancreatology. 2018;18(4):386-393.

5. Lowenfels AB, Sullivan T, Fiorianti J, Maisonneuve P. The epidemiology and impact of pancreatic diseases in the United States. Curr Gastroenterol Rep. 2005;7(2):9095.

6. Yadav D, Lowenfels AB. Trends in the epidemiology of the first attack of acute pancreatitis: a systematic review. Pancreas. 2006;33(4):323-330.

7. Baptist AP, Warrier I, Arora R, Ager J, Massanari RM. Hospitalized patients with asthma who leave against medical advice: characteristics, reasons, and outcomes. J Allergy Clin Immunol. 2007;119(4):924-929.

8. Onukwugha E, Saunders E, Mullins CD, Pradel FG, Zuckerman M, Weir MR. Reasons for discharges against medical advice: a qualitative study. Qual Saf Health Care. 2010;19(5):420-424.

9. Alfandre DJ. "I'm going home": discharges against medical advice. Mayo Clin Proc. 2009;84(3):255-260.

10. Ibrahim SA, Kwoh CK, Krishnan E. Factors associated with patients who leave acute-care hospitals against medical advice. Am J Public Health. 2007;97(12):2204-2208.

11. Fiscella K, Meldrum S, Barnett S. Hospital discharge against advice after myocardial infarction: deaths and readmissions. Am J Med. 2007;120(12):1047-1053.

12. Brook M, Hilty DM, Liu W, Hu R, Frye MA. Discharge against medical advice from inpatient psychiatric treatment: a literature review. Psychiatr Serv. 2006;57(8):11921198.

13. Hwang SW, Li J, Gupta R, Chien V, Martin RE. What happens to patients who leave hospital against medical advice? CMAJ. 2003;168(4):417-420.

14. Franks P, Meldrum S, Fiscella K. Discharges against medical advice: are race/ethnicity predictors? J Gen Intern Med. 2006;21(9):955-960.

15. Fiscella K, Meldrum S, Franks P. Post partum discharge against medical advice: who leaves and does it matter? Matern Child Health J. 2007;11(5):431-436.

16. Saitz R, Ghali WA, Moskowitz MA. The impact of leaving against medical advice on hospital resource utilization. J Gen Intern Med. 2000;15(2):103-107.

17. Weingart SN, Davis RB, Phillips RS. Patients discharged against medical advice from a general medicine service. J Gen Intern Med. 1998;13(8):568-571.

18. Myers RP, Shaheen AA, Hubbard JN, Kaplan GG. Characteristics of patients with cirrhosis who are discharged from the hospital against medical advice. Clin Gastroenterol Hepatol. 2009;7(7):786-792.

19. Chan AC, Palepu A, Guh DP, Sun H, Schechter MT, O'Shaughnessy MV, Anis AH. HIV-positive injection drug users who leave the hospital against medical advice: the mitigating role of methadone and social support. J Acquir Immune Defic Syndr. 2004;35(1):56-59.

20. Pytell JD, Rastegar DA. Who Leaves Early? Factors Associated With Against Medical Advice Discharge During Alcohol Withdrawal Treatment. J Addict Med. 2018;12(6):447-452.

21. Anis AH, Sun H, Guh DP, Palepu A, Schechter MT, O'Shaughnessy MV. Leaving hospital against medical advice among HIV-positive patients. CMAJ. 2002;167(6):633-637.

22. Kaplan GG, Panaccione R, Hubbard JN, Nguyen GC, Shaheen AA, Ma C, Devlin SM, et al. Inflammatory bowel disease patients who leave hospital against medical advice: predictors and temporal trends. Inflamm Bowel Dis. 2009;15(6):845-851.

23. Phillips DP, Christenfeld N, Ryan NM. An increase in the number of deaths in the United States in the first week of the month - an association with substance abuse and other causes of death. N Engl J Med. 1999;341(2):9398.

24. Halpern SD, Mechem CC. Declining rate of substance abuse throughout the month. Am J Med. 2001;110(5):347351.

25. Saitz R. Discharges against medical advice: time to address the causes. CMAJ. 2002;167(6):647-648.

26. Alebiosu CO, Raimi TH. Discharge against medical advice. Trop Doct. 2003;33(3):191-192.

27. Andrade SE, Gurwitz JH, Chan KA, Donahue JG, Beck A, Boles M, Buist DS, et al. Validation of diagnoses of peptic ulcers and bleeding from administrative databases: a multi-health maintenance organization study. J Clin 
Epidemiol. 2002;55(3):310-313.

28. Fagenholz PJ, Fernandez-del Castillo C, Harris NS, Pelletier AJ, Camargo CA, Jr. Direct medical costs of acute pancreatitis hospitalizations in the United States. Pancreas. 2007;35(4):302-307.

29. Garg SK, Campbell JP, Anugwom C, Wadhwa V, Singh
R, Gupta N, Sanaka MR. Incidence and predictors of readmissions in acute pancreatitis: a nationwide analysis. Pancreas. 2018;47(1):46-54.

30. Wadhwa V, Patwardhan S, Garg SK, Jobanputra Y, Lopez R, Sanaka MR. Health care utilization and costs associated with acute pancreatitis. Pancreas. 2017;46(3):410-415. 